\title{
TWODIMENSIONAL AXIALSYMMETRICAL HYDRODYNAMICAL SIMULATIONS OF PN-EVOLUTION
}

\author{
J. ZWEIGLE and M. BREMER \\ Astronomisches Institut der Universität Waldhäuserstrasse 64, D-7400 Tübingen, \\ Germany \\ M. GREWING \\ Astronomisches Institut der Universität Waldhäuserstrasse 64, D-7400 Tübingen, \\ Germany \\ and
}

Institut de Radioastronomie Millimtrique (IRAM), 300 Rue de la Piscine, F-38406 Saint Martin d'Hères

In order to investigate the early evolution of planetary nebulae $(\mathrm{PNe})$ we solved numerically the hydrodynamical equations in cylindrical coordinates $(r, z)$ assuming azimutal symmetry. The numerical method used is described in detail by Mair et al. (1988). Our simulations model the interaction of a fast, tenuous, spherical symmetrical central star wind with a slow, dense, aspherical Red Giant Envelope (RGE) expelled from the progenitor star. For the aspherical RGE with a polar/equatorial density contrast we used the initial model given by Mellema et al. (1991) in cylindrical coordinates. We have investigated the influence of each initial model parameter upon the evolution of PNe. Thereby we confirm that the polar/equatorial density contrast in the RGE and the thickness of the RGE-disk play an important role for the morphology of PNe. In agreement with the results from Mellema et al. (1991). The polar/equatorial density contrast in the RGE influences the ratio of the distances of the bright inner rim to the central star in $\mathrm{z}$ - and $\mathrm{r}$-direction. This ratio increases with decreasing polar/equatorial density contrast. We find the thickness of the RGE-disk to be a key parameter for getting an elliptical or a butterfly PN: thin RGE-disks produce the first type of nebulae, thick disks the latter. We thank G. Mair, E. Müller and W. Hillebrandt for making available to us a copy of the SADIE code.

\section{References}

Mair, G. et al. 1988, Astron. Astrophys. 199,114

Mellema G. et al. 1991, Astron. Astropys. 252,718 